\title{
Evaluation of the retinal nerve fiber layer thickness and the choroidal thickness in generalized vitiligo patients
}

\author{
Müslüm Toptan ${ }^{1}$ and Mustafa Aksoy $^{1}$ \\ ${ }^{1}$ Harran Universitesi
}

July 1, 2020

\begin{abstract}
Aim: The retinal nerve fiber layer (RNFL) thickness and the choroidal thickness is compared to healthy volunteers in vitiligo patients. Material and method: The study included the right eyes of 60 healthy individuals and 60 generalized vitiligo patients. Following routine ophthalmological examination, the RNFL thickness and the choroidal thickness were analyzed with spectral domain optical coherence tomography (SD-OCT). Results: It was determined that the mean macular choroidal thickness decreased significantly in vitiligo patients, while the RNFL thickness did not change $(\mathrm{p}<0.05)$. Conclusion: As the fact that the RNFL thickness does not change despite the significantly reduced choroidal thickness in vitiligo patients may indicate that the melanin cells that are dense in the choroid are affected, the non-invasive, simple and rapid measurement of the choroidal thickness with OCT can help in determining the damage in vitiligo patients. Keywords: Vitiligo, choroid, nerve fibers, optical coherence tomography. 1. What is already known about this topic? Melanocytes are found in uveal tissues such as the eyelash, retinal pigment epithelium, choroid, ciliary body, and iris. Melanin in these tissues can disappear when destruction occurs in cutaneous melanocytes in vitiligo patients. As a result, some studies reported hypopigmented spots on the iris and retina, atrophic changes in the peripapillary area, degeneration in the retinal pigment epithelium and chorioretinal areas. Melanin, produced in melanocytes in the choroidal layer and stored in melanosomes, has a crucial role in protecting and absorbing light from intraocular reflection. 2. What does this article add? Although the visual acuity of the patients with vitiligo is at the same level as the control group, we found that the average macular choroid thickness was thinner. We found no significant changes in the choroidal thickness in patients with periocular attitude. We found a negative correlation between the choroidal thickness and the duration of the disease.
\end{abstract}

\section{Introduction}

Vitiligo is a disease whose etiopathogenesis is unknown, characterized by the formation of depigmentation in different parts of the skin due to melanocyte destruction (1). The loss of pigment cells may not be limited to the skin, and various ocular abnormalities can be seen as melanocytes in the eyes develop from neural crest cells $(2,3,4)$. These melanocytes are found in uveal tissues such as the eyelash, retinal pigment epithelium, choroid, ciliary body, and iris. Melanin in these tissues can disappear when destruction occurs in cutaneous melanocytes in vitiligo patients (5). As a result, some studies reported hypopigmented spots on the iris and retina, atrophic changes in the peripapillary area, degeneration in the retinal pigment epithelium and chorioretinal areas $(2,3,6)$.

The choroid is a highly vascularized and pigmented tissue that feeds the outer retina, and especially the stroma of the choroidal layer contains a high number of melanocytes. Melanin, produced in melanocytes in the choroidal layer and stored in melanosomes, has a crucial role in protecting and absorbing light from intraocular reflection (4). There are a limited number of studies showing that the affected choroid is also affected in vitiligo in some systemic inflammatory diseases $(5,7)$.

Rogosic et al. found that there was an association between the duration of vitiligo and the development of 
glaucoma (8).

There are also studies indicating impaired retinal electrophysiological function in vitiligo patients $(9,10)$. Abnormal electro-oculographic findings of visual evoked potentials were observed, especially in patients with vitiligo with large skin involvement and longer disease duration (10).

We aimed to compare the choroid and the RNFL thicknesses of vitiligo patients using spectral domain optical coherence tomography (SD-OCT) because it is not known enough how and how much the sensitive retinal nerve fiber layer (RNFL) of specific importance is affected in the evaluation of glaucomatous damage with the highly pigmented and vascularized choroidal tissue.

\section{Material and method}

This study was conducted in the Department of Eye Diseases and Skin and Venereal Diseases of Harran University in 2019-2020. Study protocol was approved by the Ethics Committee of Harran University (Document Number: E.35564) and informed consent was received from the participants. The right eyes of sixty generalized vitiligo patients and the right eyes of 60 healthy individuals paired with this patient group in terms of age and gender were included in the study as a control group.

A comprehensive medical history including the drugs used for each patient was taken and general dermatological physical examinations were performed by the same dermatologist. Both groups also underwent a full ophthalmological examination, which included the best corrected visual acuity, intraocular pressure measurement with the Goldmann Applanation Tonometer, refraction, biomicroscopy of the anterior and posterior segment of the eye. Axial length was measured using ultrasonic biometrics Pac-Scan 300p (Sonomed Escalon, NY) and visual field examination was performed using Humphrey perimetry (Carl Zeiss Meditec, Dublin, CA) with 30-2 program.

People under the age of 18, people with cataract or glaucoma, people with a BCVA of 20/40, people with a history of ocular surgery, people with optic nerve or retina disorders, people with systemic diseases that can affect the eye such as diabetes mellitus and hypertension were not included in the study and all patients between the ages of 18 - 40 with generalized vitiligo diagnosis were included in the study.

Choroidal thickness measurements were performed by the same experienced technician in increased depth imaging mode with the Heidelberg spectral domain optical coherence tomography device (SD-OCT, Heidelberg Engineering, Germany). The choroidal thickness was measured manually by marking the distance between the outer border of the retinal pigment epithelium and the choroid-sclera compound. In addition to the thickness of the subfoveal choroid, seven measuring points were determined, including 3 nasal and 3 temporal with $500 \mu \mathrm{m}$ intervals in the temporal and nasal directions. N1, N2 and N3 abbreviations were given to the points identified in the nasal respectively, from the central to the periphery; and T1, T2 and T3 abbreviations were given to the points identified in the temporal respectively, from the central to the periphery (figure 1). Peripapillary RNFL thickness parameters were calculated automatically with fast RNFL mode. The software calculated average thickness values $(\mu \mathrm{m})$ for each of the six sectors centred on the optical disk (temporal, temporal superior, temporal inferior, nasal, nasal inferior, and nasal superior) (figure 2). Sections below signal strength index 6/10 were not evaluated. Measurements were made between 09.00-12.00 in the morning to prevent diurnal changes.

\section{Statistical analysis}

Statistical evaluation was conducted with the IBM SPSS 15 package program (SPSS Inc., Chicago, IL, USA). Numerical variables with normal distribution are given as mean $+/-$ standard deviation, numerical variables without normal distribution are given as median (minimum-maximum), and categorical variables as frequency (percentages). In the case of two groups, the difference between the groups was determined with the Student $t$ Test for the numerical variables with normal distribution, the Mann Whitney U Test for the numerical variables without normal distribution, the Pearson Chi-Square Test and the Fischer Exact Test. The relationship between numerical variables was determined with the Pearson correlation analysis. $\mathrm{P}$ value of 0.05 was considered statistically significant. 


\section{Results}

The demographic characteristics and clinical findings of the individuals in the group are shown in Table 1. There is no statistically significant difference between the patient and the control group in terms of age, sex, IOP (intraocular pressure), axial length, MD (mean deviation), PSD (patent standard deviation) and visual acuity variables $(\mathrm{p}>0.05)$.

Ocular fundus findings such as peripapillary atrophy in three patients, focal hypopigmentation in two patients and widespread hypopigmentation in one patient were found.

The mean choroidal thickness was $275,42(202,57-379,14) \mu \mathrm{m}$ in the patient group and 297,50 (182,71-371,71) $\mu \mathrm{m}$ in the control group $(\mathrm{p}=0.006)$.

While there was a statistically inversely low association between subfoveal choroidal thickness and duration of disease in vitiligo $(\mathrm{p}=0,029, \mathrm{r}=-0,283)$, there was no statistically significant association between age and the choroidal thickness $(\mathrm{p}>0.05)$ (Table 2$)$.

While there was statistically significant difference between the patient and the control group in terms of T3, $\mathrm{T} 2$, T1, subfoveal, N1 and mean values $(\mathrm{p}<0.05)$, there was no statistically significant difference between N2 and N3 values $(\mathrm{p}>0.05)$ (Table 3$)$.

While there was statistically significant difference between the patients and the control group in terms of superionasal and inferiotemporal values $(\mathrm{p}<0.05)$, there was no statistically significant difference between the groups in terms of global, temporal, superiotemporal, nasal, and inferionasal values ( $\mathrm{p}>0.05)$ (Table 4).

While there was no statistically significant difference between periocular involvement and non-macular choroidal values in the patient group, macular choroidal thickness of periocular involvement patients was observed to be thinner (Table 5).

There was no statistically significant difference in the RNFL thickness between those with periocular involvement and those without (Table 6).

\section{Discussion}

Although many factors have been blamed for the pathogenesis of vitiligo, the etiology is not yet fully known. Antibodies against melanocytes are known to be destructive to melanocytes in the vast majority of patients (1).

Evidence of the destruction of uveal melanocytes and retinal pigment epithelium in vitiligo patients was first reported by Albert et al (11). In some studies, hypopigmentation of the choroid was also detected in vitiligo patients $(12,13)$. Bülbül et al. , in their study which examined ocular findings in 45 vitiligo patients, found peripapillary atrophy around the optic nerve in seven patients, atrophy of the retina pigment epithelium in two patients, diffuse in the retina in one patient, focal hypopigmented spots in one patient. Their study also found that ocular findings were significantly observed in patients with periorbital and to a lesser degree genital vitiligo (6). Biswas et al. found pigment change in iris and anterior chamber in 41 of 100 vitiligo patients, retinal pigment epithelial hypopigmentation in 9 of them, uveitis in 5 of them, chorioretinal degeneration in 11 of them, and reported no ocular findings in 34 of them, indicating a strong association between vitiligo and ocular disorder (3). Karadağ et al. found abnormal retinal findings in 24 out of 122 eyes in 122 vitiligo patients; determined Tigroid retina in 11 eyes, oblique disc in 3 eyes, peripapillary atrophy in 3 eyes, drusen in 2 eyes, choroidal nevus in 1 eye, myelinated nerve fiber in 1 eye, cage degeneration in 1 eye (2). In our study, we found ocular fundus findings such as peripapillary atrophy in three patients, focal hypopigmentation in two patients and widespread hypopigmentation in one patient.

Studies on the choroidal tissue in vitiligo patients have been limited, especially in-vivo study of the choroid with the EDI form of the SD-OCT device has helped to understand the diagnosis and pathogenesis of some diseases more clearly. The choroidal thickness is known to be affected by age, sex and ax (14). In our study, there were no significant differences between age, gender, and ax among the groups. 
The choroid is among the most vascularized tissues in the body, covering the outer retina. A pathology that can occur in the choroidal structure for any reason can result in decreased vision as a result of degenerative changes in photoreceptors. The stroma of the choroidal layer contains a high number of melanocytes (4). A low choroidal thickness may be expected in vitiligo where melanocyte loss is observed. Foveal hypoplasia was found in several studies in patients with albinism $(15,16)$. In patients with oculocutaneous albinism with melanocyte deficiency, the choroidal thickness in the subfoveal region was significantly lower than in the control group and there was no difference in the peripapillary region compared to the control group (17). Demirkan et al. determined that the choroidal thickness in vitiligo patients was significantly thin compared to the control group in all values except optic nerve area measurements, and it was indicated that choroidal thickness may be affected by melanocyte loss in vitiligo. The absence of differences between vitiligo patients and the control group in optic nerve regions was linked to less space coverage of melanocytes in histological structure in optic nerve regions. It was also stated that periorbital involvement had no effect on the choroidal thickness (18).

Vogt-Koyanagi-Harada (VKH) syndrome is a disease characterized by a T cell-mediated autoimmune process aimed at melanocytic antigens. Ocular symptoms of the disease include severe bilateral panuveitis associated with exudative retinal detachment. Retinal edema, choroidal thickening, bilateral vitritis, papillitis and retinal detachment are seen. As exudation is not observed in vitiligo patients, increase in the choroidal thickness is not expected despite melanocyte destruction as in VKH (20). We also determined that even though visual acuity of vitiligo patients was at the same level as the control group, the mean macular choroidal thickness was thinner. We found no significant changes in the choroidal thickness in patients with periocular attitude. We found a negative correlation between the choroidal thickness and the duration of the disease. Also, in our study, although there was significant decrease in the choroidal thickness of N1, T1, T2, T3 in vitiligo patients, there was no significant change in $\mathrm{N} 2, \mathrm{~N} 3$, and this may indicate that the choroidal thickness changed slightly in this region due to the less dense melanin cells around the optic disc.

In the diagnosis of glaucoma, the determination of thickness thinning of OCT and RNFL is considered the most specific test for objective detection of damage (21). In the studies of Rogosic et al., the age of the patient and the duration of vitiligo were significantly correlated with primary open-angle glaucoma. In their study, the RNFL thickness was measured to gauge neuroretinal damage (8).

Dertlioglu et al. reported in their studies that normotensive glaucoma was seen in $18.4 \%$ of vitiligo group and that there was a statistically significant difference compared to control group. They found no correlation between periocular involvement and glaucoma (22). Duplancić et al. reported that the prognostic value of hemodynamic changes determined by color Doppler imaging in the ophthalmic artery and that there was a positive correlation between vitiligo and POAG (23). In many sources, it was that glaucoma seen in vitiligo is a secondary complication, a direct result of corticosteroid therapy $(24,25)$. Studies of Khurrum et al. demonstrated that patients with vitiligo and periorbital topical steroids did not have an increased risk of glaucoma or cataracts (26). Örnek et al., in their study, reported no significant differences between vitiligo patients and control groups in the upper, lower, nasal and temporal quadrants in the topographic distribution of RNFL, and concluded that the RNFL thickness was not affected in vitiligo patients (5).

Electroretinography (ERG) and visual evoked potentials (VEP), which are electrophysiological tests, are useful tests for determining retinal ganglion cell and RNFL dysfunction (27). Shoeibi et al. reported that overall retinal electrophysiological function in vitiligo patients decreased significantly compared to the normal population regardless of age and sex (9). Perossini et al. identified abnormal VEP and electrooculography (EOG) in patients with severe vitiligo. They found more pronounced negative ocular electrophysiological results in patients with greater skin involvement and longer duration of disease (10). In an animal study, abnormal flash ERG findings secondary to histopathological abnormalities were reported in mutant mice with vitiligo, and the apopitosis of photoreceptors in these mice was demonstrated in morphological and biochemical studies (28). Aydin et al., in their studies on vitiligo patients, found that the photoreceptor segment was preserved in "SD-OCT", while in multifocal electroretinography (mfERG), there was a decrease in central retinal function, indicating a potential decrease. This study showed that even with normal fundus 
appearance and SD-OCT findings, there may be a potential decline in central retinal function in vitiligo patients. Their study also noted that there was no statistically significant difference in the entire retinal layer thickness between the vitiligo and the control group (29).

Since the choroidal thickness is affected by many factors and diurnal choroidal thickness changes occur at $30-60 \mu \mathrm{m}(14)$, we found it appropriate to measure the choroidal thickness values in a limited time period (between 09: 00 and 12:00 hours). Low case count, short follow-up time, the fact that factors which can affect the choroidal thickness measurements such as smoking and caffeine use are not studied, and manual measurement of the choroidal thickness are limited aspects of our study. Studies with a longer followup period and broader participation investigating all factors that may affect the choroidal and the RNFL thickness in vitiligo patients may provide greater clarity on this issue.

As far as we know, this study is the first to examine the choroidal thickness and the RNFL thickness with SD-OCT in vitiligo patients, analyzing its correlation with disease duration. In our study, it was determined that mean macular choroidal thickness decreased significantly in vitiligo patients, while the RNFL thickness did not change. Negative correlation was found between the choroidal thickness and the duration of the disease. There was no significant correlation between the choroidal and the RNFL thickness in vitiligo patients with periocular involvement. We are of the opinion that close monitoring of the vitiligo patients by the eye diseases department, the non-invasive OCT screening for posterior ocular segment disorders, and OCT-EDI examinations for the choroidal thickness containing dense melanin may be much more beneficial.

\section{References}

1. Schild M, Meurer M. [Vitiligo: Clinical presentation and pathogenesis]. Hautarzt. 2016 Feb;67(2):173-86; quiz 187-9.

2. Karadag R, Esmer O, Karadag AS, Bilgili SG, Cakici O, Demircan YT, et al. Evaluation of ocular findings in patients with vitiligo. Int J Dermatol. 2016 Mar;55(3):351-5.

3. Biswas G, Barbhuiya JN, Biswas MC, Islam MN, Dutta S. Clinical pattern of ocular manifestations in vitiligo. J Indian Med Assoc. 2003 Aug;101(8):478-80.

4. Cook CS, Ozanis V, Jakobiec FA. Prenatal development of the eye and its adnexa. In: Tasman W (ed.) Duane's Ophthalmology. Lippincot-Raven Publishers, Inc: Philadelphia, 1991, pp 1-93.

5. Ornek N, Onaran Z, Koçak M, Ornek K. Retinal nerve fiber layer thickness in vitiligo patients. J Res Med Sci. 2013 May;18(5):405-7.

6. Bulbul Baskan E, Baykara M, Ercan I, Tunali S, Yucel A. Vitiligo and ocular findings: a study on possible associations. J Eur Acad Dermatol Venereol. 2006 Aug;20(7):829-33.

7. Kurt A, Kurt EE, Kilic R, Oktem C, Tuncay F, Erdem HR. Is choroidal thickness related with disease activity and joint damage in patient with rheumatoid arthritis. Bratisl Lek Listy. 2017; 118 (1): 23-7.

8. Rogosić V, Bojić L, Puizina-Ivić N, Vanjaka-Rogosić L, Titlić M, Kovacević D, et al. Vitiligo and glaucoma - an association or a coincidence? A pilot study. Acta Dermatovenerol Croat. 2010;18(1):21-6. Erratum in: Acta Dermatovenerol Croat. 2010 Jul;18(2):119.

9. Shoeibi N, Taheri AR, Nikandish M, Omidtabrizi A, Khosravi N. Electrophysiologic evaluation of retinal function in patients with psoriasis and vitiligo. Doc Ophthalmol. 2014 Apr;128(2):131-6.

10. Perossini M, Turio E, Perossini T, Cei G, Barachini P. Vitiligo: ocular and electrophysiological findings. G Ital Dermatol Venereol. 2010 Apr;145(2):141-9.

11. Albert DM, Nordlund JJ, Lerner AB. Ocular abnormalities occurring with vitiligo. Ophthalmology. 1979 Jun;86(6):1145-60.

12. Kim M, Kwon JW, Park YH. Atypical Pattern of Choroidal Hypopigmentation with Cutaneous Vitiligo. Korean J Ophthalmol. 2019 Feb;33(1):99-100. 
13. Ciardella AP, Horsley MB, Brown DM. Hypopigmentary fundus changes seen with cutaneous vitiligo. Arch Ophthalmol. 2007 Apr;125(4):576. Erratum in: Arch Ophthalmol. 2007 Jun;125(6):849.

14. Aksoy Y, Çolakoglu K, Kar T, Sevinc MK, Eyi YE. Choroidal thickness is affected by more factors than estimated. Invest Ophthalmol Vis Sci. 2014 Mar 4;55(3):1311.

15. Thomas MG, Kumar A, Mohammad S, Proudlock FA, Engle EC, Andrews C, et al. Structural grading of foveal hypoplasia using spectral-domain optical coherence tomography a predictor of visual acuity? Ophthalmology. 2011 Aug;118(8):1653-60.

16. Seo JH, Yu YS, Kim JH, Choung HK, Heo JW, Kim SJ. Correlation of visual acuity with foveal hypoplasia grading by optical coherence tomography in albinism. Ophthalmology. 2007 Aug;114(8):1547-51.

17. Karabas L, Esen F, Celiker H, Elcioglu N, Cerman E, Eraslan M, et al. Decreased subfoveal choroidal thickness and failure of emmetropisation in patients with oculocutaneous albinism. Br J Ophthalmol. 2014;98:1087-90.

18. Demirkan S, Onaran Z, Samav G, Özkal F, Yumuşak E, Gündüz Ö, et al. Decreased choroidal thickness in vitiligo patients. BMC Ophthalmol. 2018 May 29;18(1):126.

19. Street D, Sivaguru A, Sreekantam S, Mollan SP. Vogt-Koyanagi-Harada disease. Pract Neurol. 2019 Aug;19(4):364-367.

20. Perente I, Utine CA, Cakir H, Kaya V, Tutkun IT, Yilmaz OF. Management of ocular complications of Vogt-Koyanagi-Harada syndrome. Int Ophthalmol. 2009 Feb;29(1):33-7. Epub 2007 Oct 19.

21. Zangwill LM, Bowd C. Retinal nerve fiber layer analysis in the diagnosis of glaucoma. Curr Opin Ophthalmol. 2006 Apr;17(2):120-31.

22. Dertlioglu SB, Oguz H, Cicek D, Yücel H. Prevalence of glaucoma in patients with vitiligo. Cutis 2016;97: $21-25$.

23. Duplancić D, Rogosić V, Puizina-Ivić N, Rogosić LV, Luksić B, Kovacić V, et al. [Prognostic value of ophthalmic artery color Doppler sonography for progression to glaucoma in vitiligo patients]. Acta Med Croatica. 2013 Mar;67(1):47-52.

24. Daniel BS, Orchard D. Ocular side-effects of topical corticosteroids: what a dermatologist needs to know. Australas J Dermatol. 2015 Aug;56(3):164-9.

25. David DS, Berkowitz JS. Ocular effects of topical and systemic corticosteroids. Lancet. 1969 Jul 19;2(7612):149-51.

26. Khurrum H, Alhamdi KM, Osman E. Screening of glaucoma or cataract prevalence in Vitiligo patients and its relationship with periorbital steroid use. J Cutan Med Surg. 2016 Mar-Apr; 20 (2): 146-9.

27. Kurysheva NI, Maslova EV, Zolnikova IV, Fomin AV, Lagutin MB. A comparative study of structural, functional and circulatory parameters in glaucoma diagnostics. PLoS One. 2018 Aug 23;13(8):e0201599.

28. Tang M, Pawlyk BS, Kosaras B, Berson EL, Sidman RL. ERG abnormalities in relation to histopathologic findings in vitiligo mutant mice. Exp Eye Res. 1997 Aug;65(2):215-22.

29. Aydin R, Ozsutcu M, Erdur SK, Dikkaya F, Balevi A, Ozbek M, et al. The assessment of macular electrophysiology and macular morphology in patients with vitiligo. Int Ophthalmol. 2018 Feb;38(1):233239.

\section{Hosted file}

IJCP.docx available at https://authorea.com/users/338733/articles/465193-evaluation-of-theretinal-nerve-fiber-layer-thickness-and-the-choroidal-thickness-in-generalized-vitiligopatients 


\section{Hosted file}

table all.docx available at https://authorea.com/users/338733/articles/465193-evaluationof-the-retinal-nerve-fiber-layer-thickness-and-the-choroidal-thickness-in-generalizedvitiligo-patients

\section{Hosted file}

Figures.docx available at https://authorea.com/users/338733/articles/465193-evaluationof-the-retinal-nerve-fiber-layer-thickness-and-the-choroidal-thickness-in-generalizedvitiligo-patients 